\title{
Chaotic Signal for Signal Masking in Digital Communications
}

\author{
Rahul Ekhande ${ }^{1}$, Sanjay Deshmukh ${ }^{2}$ \\ ${ }^{\text {I}(M . E ~ S t u d e n t, ~ D e p t . ~ o f ~ E l e c t r o n i c s ~ \& ~ T e l e c o m m u n i c a t i o n s, ~ R a j i v ~ G a n d h i ~ I n s t . ~ O f ~ T e c h n o l o g y, ~ M u m b a i ~}$ \\ University, India) \\ ${ }^{2}$ (Asst. Professor, Dept. of Electronics \& Telecommunications, Rajiv Gandhi Inst. Of Technology, Mumbai \\ University, India)
}

\begin{abstract}
A signal masking technique based on Lorentz System is presented in this paper which uses Lorentz equation generated Chaotic Signals are used as a base carrier signal for information signal modulation in the transmitter system. The scheme recovers the information signal exactly at the receiver. The mixing property of the implemented scheme investigated by Computer Simulations shows better performance than that of the conventional method
\end{abstract}

Keywords: - Chaotic Signal, Lorentz System, Lorentz Equations, Attractor, Lyapunov Exponent

\section{INTRODUCTION}

Conventionally, a pseudo random $(\mathrm{PN})$ sequence was used for direct-sequence code division multiple access (DS-CDMA) systems, but it does not security due to fact that there are restricted number of available PN sequences and they show periodic correlation properties. Chaotic sequences, based on chaotic studies, are nonbinary and non-periodic sequences [1]. As the number of available chaotic signals is large it is very difficult for an interceptor to crack the chaotic sequence even if a chaotic function is known. This provides huge advantages over the conventional PN sequences based systems. Chaotic systems provide a rich mechanism for signal design and generation, with potential applications to communications and signal processing. Because chaotic signals are typically broadband, noise-like, and difficult to predict, they can be used in various contexts in communications.

A particularly useful class of chaotic systems is those that possess a self-synchronization property [2]. This property allows two identical chaotic systems to synchronize when the second system (receiver) is driven by the first (transmitter). Arneodo Attractor's chaotic oscillator circuits were designed and simulated successfully [3].This paper implements Lorentz Chaotic System and demonstrates simulations that chaos can be synchronized and applied to signal masking communications are organized into five parts. Following this introduction, part II provides the detail DS-CDMA system. Part III will provide information about Lorentz System and its chaotic nature with chaotic signal generation which will be used as carrier signal for Information signal encryption. Part IV provides the simulation of Information signal modulation and demodulation with chaotic signal at transmitter and receiver respectively. Part V provides the concluding remarks. Part VI \& VII end the paper by Acknowledgement and References respectively

\section{DS-CDMA SYSTEM}

Spread spectrum signals for digital communications were originally invented for military communication, but nowadays they are widely used to provide reliable communication in a variety of commercial applications including mobile and wireless communications, which provide resistance to hostile jamming, hide the signal by transmitting it at low power, or make it possible for multiple users to communicate through the same channel. Conventionally binary pseudo-random (PN) sequence codes were used. It is a deterministic, periodic signal that is known to both transmitter and receiver which appears to an unauthorized listener, to be similar to white noise. DS-CDMA is a multiple access technique in which multiple users can transmit their data on the same channel using orthogonal spreading sequences [4].

\section{THE LORENTZ SYSTEM}

The Lorentz system of differential equations arose from the work of Edward N. Lorentz [5]. He came with three differential equations which produce vast different solutions for small differences in initial conditions, a characteristic as Chaos [2]. The system of differential equations of Lorentz used was

$$
\text { ......Eq. 1(a) }
$$




$$
\begin{gathered}
\dot{x}=-\sigma x+\sigma y \\
\dot{y}=r x-y-x z \\
\dot{z}=-b z+x y
\end{gathered}
$$

where sigma; $r$; and $b$ are positive parameters. The Figure1and Figure2 shows the Simulink modeling and the simulation results of the Lorentz Attractor respectively. With sigma $=10 ; r=28 a n d \quad b=8 / 3$. The series does not form limit cycles nor does it ever reach a steady state. Instead it is an example of deterministic chaos. As with other chaotic system the Lorentz system is sensitive to initial conditions, two initial states no matter how close will diverge, usually sooner rather than later.[5]

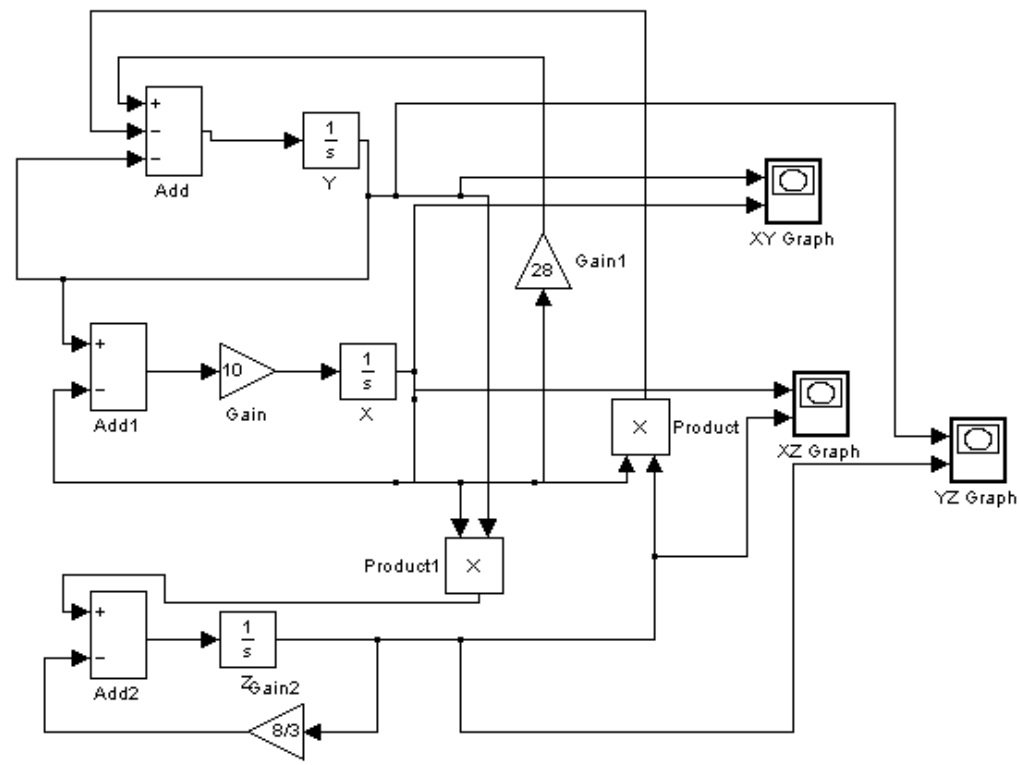

Figure 1. Matlab-Simulink Model of the Lorentz Attractor

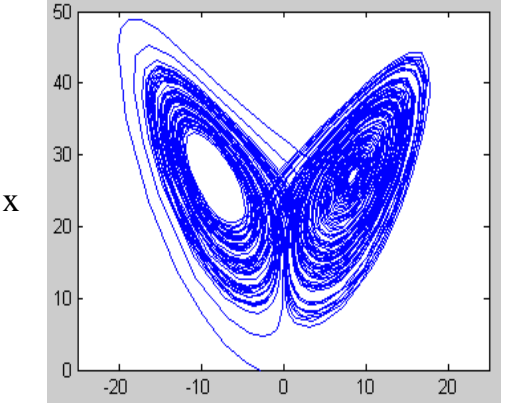

$\mathrm{y}$

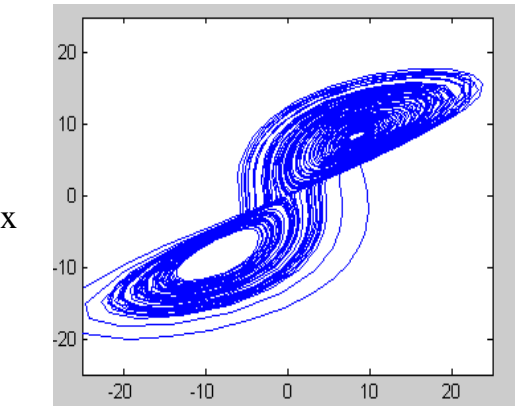

z

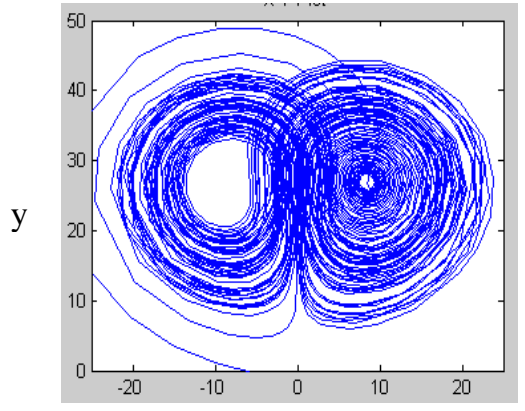

Z

Figure 2. Phase portraits ((a) x-y, (b) x-z, (c) y-z) of the Lorentz Attractor

Study of above simple, interesting, and yet complex three-dimensional quadratic autonomous chaotic system which can generate complex 2-scroll chaotic attractors simultaneously having unknown dynamical behaviors of this chaotic attractor are desirable for engineering applications such as secure communications [10].The chaotic nature of signal depends on the Lyapunov exponent.

The Lyapunov exponent measures the sensitivity of a dynamical system to small changes in initial conditions, and is used primarily for identifying chaos. If two orbits start close to one another in a chaotic system, then they will tend to move away from each other exponentially. We can write this idea as

$$
\frac{d}{d_{0}}=e^{\lambda\left(t-t_{0}\right)} \quad \text {......Eq. } 2
$$

where $\mathrm{d} 0$ is the initial displacement between a starting point and a nearby neighbor at initial time t0. The variable $\mathrm{d}$ is the displacement at time $\mathrm{t}>\mathrm{t} 0$. The $\lambda$ is the Lyapunov exponent. We can see that if $\lambda>0$, then $\mathrm{d} /$ 
$\mathrm{d} 0$ grows exponentially with the passage of time; if $\lambda<0$, then $\mathrm{d} / \mathrm{d} 0$ shrinks to zero. If $\lambda=0$, then the change in displacement over time is non-exponential. [12]

$\lambda \quad>0$ system is chaotic

$\lambda<0 \ldots$ system is non-chaotic

\section{CHAOTIC SIGNAL MASKING CIRCUITS}

The presence of the chaotic signal between the transmitter and receiver has proposed the use of chaos in secure communication systems Figure 3. The design of these systems depends on the self-synchronization property of the Lorentz Attractor [2]. Transmitter and receiver systems are identical except as shown in Figure.3. It is necessary to make sure the parameters of transmitter and receiver are identical for implementing the chaotic masking communication.

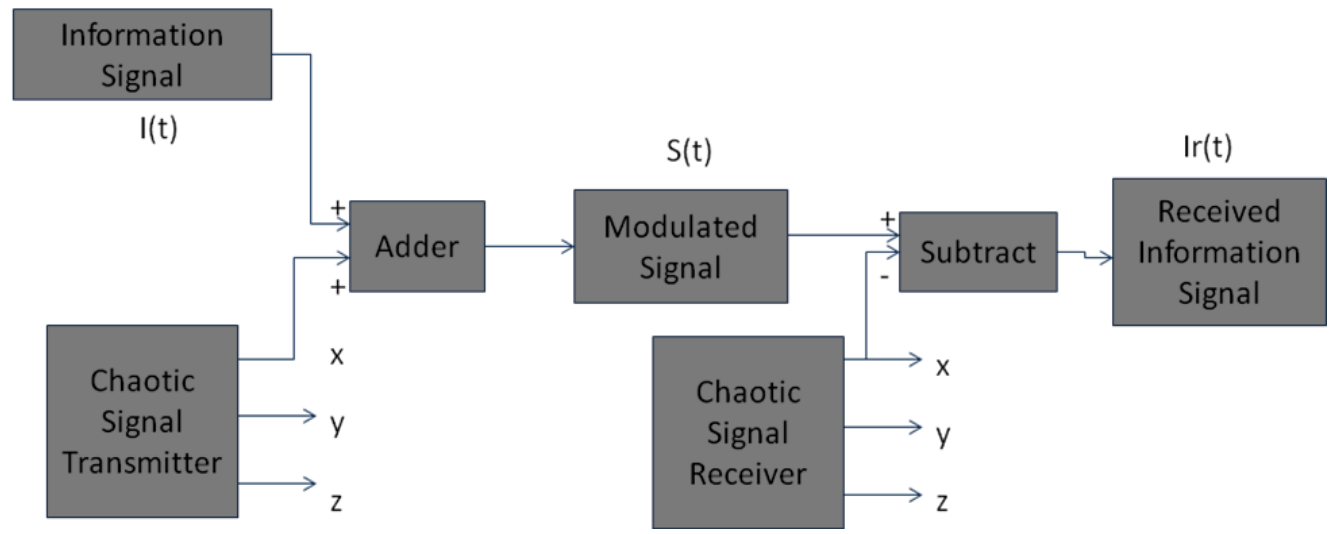

Figure 3. Block Diagram of Chaotic Communication

In this masking scheme, a low-level message signal is added to the synchronizing driving chaotic signal in order to regenerate a clean driving signal at the receiver. Thus, the message has been perfectly recovered by using the signal masking approach through cascading synchronization in the Lorentz Attractor.

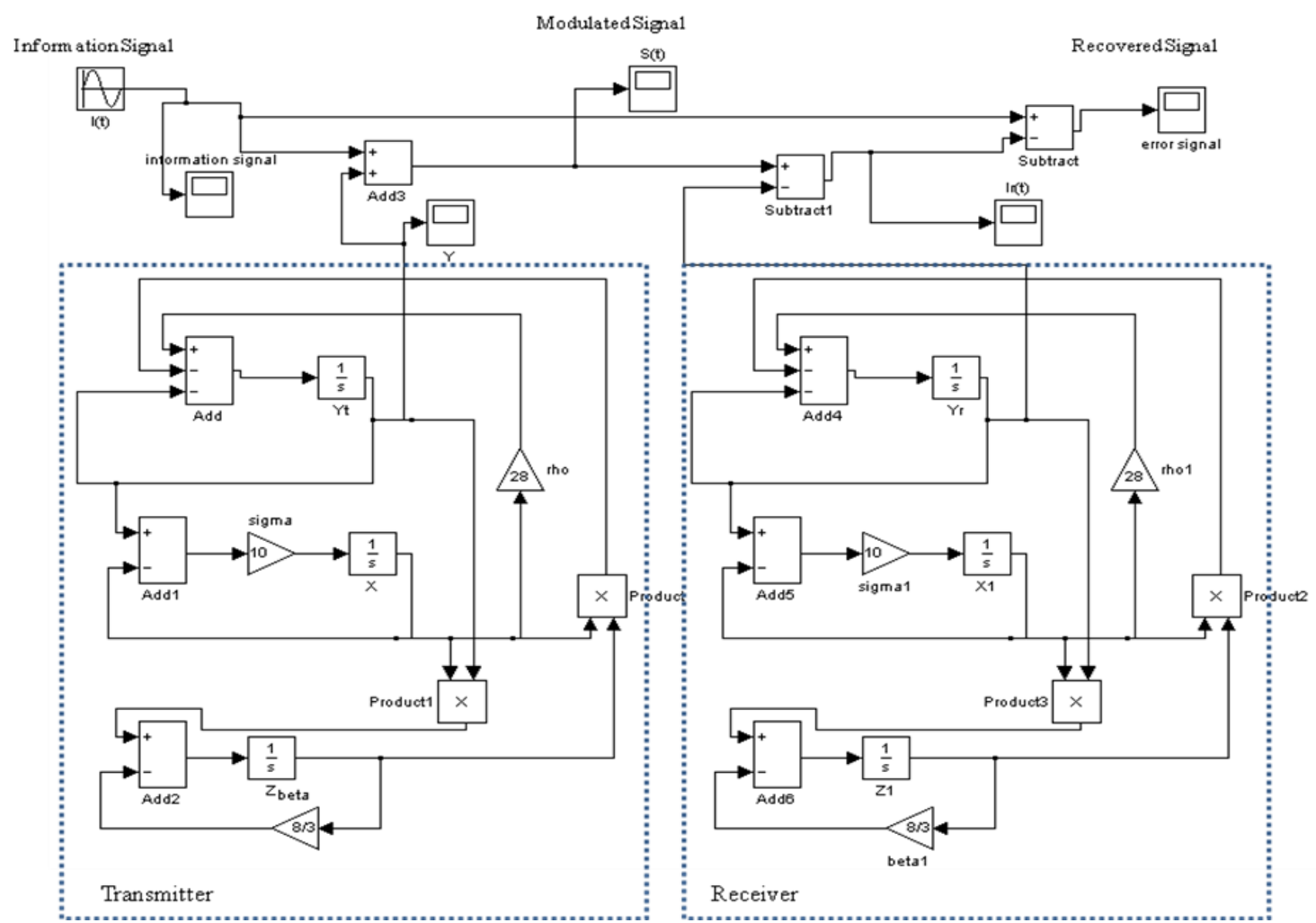

Figure 4. Phase portraits ((a) $x-y,(b) x-z,(c) y-z)$ of the Lorentz Attractor 


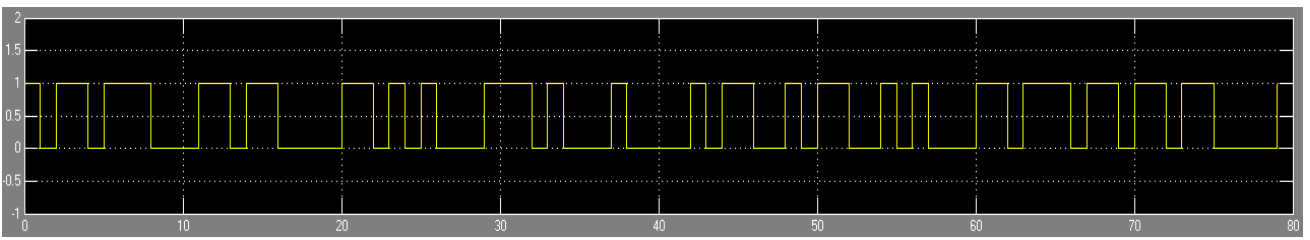

Figure 5

Time

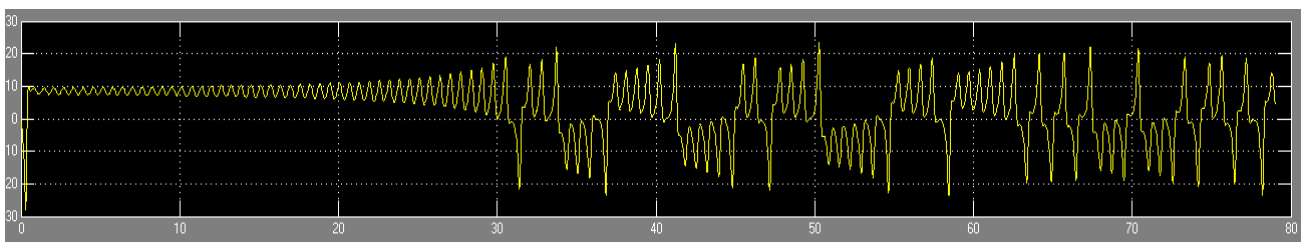

Figure 6 Time

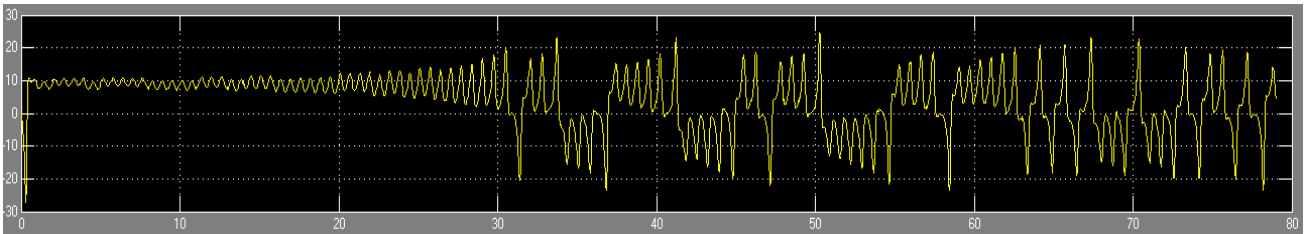

Figure 7. Time

Amplitude

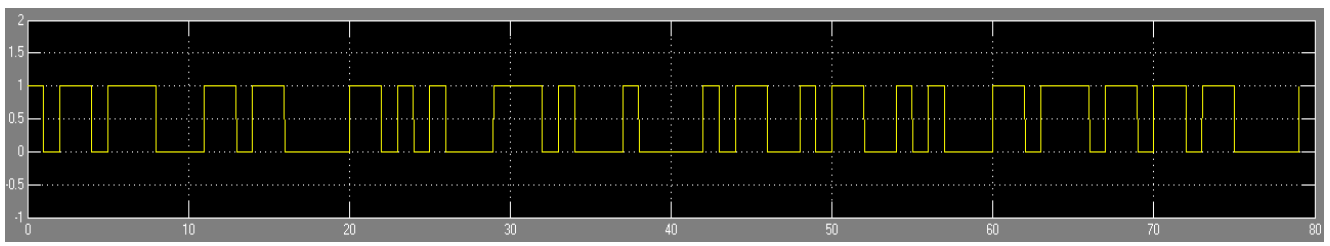

Figure 8 Time

Amplitude

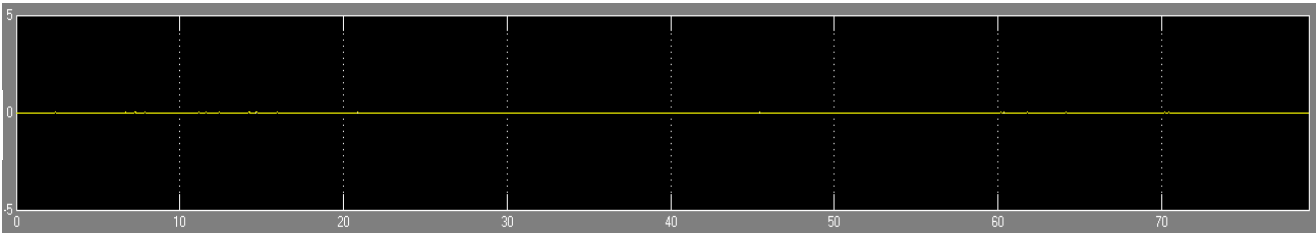

Figure 9 Time

Figure 5. Information Signal I (t)

Figure 6. Transmitter Chaotic Signal y

Figure 7. Modulated Signal S (t)

Figure 8. Recovered Information Signal Ir (t)

Figure 9. Error Signal e(t)

The transmitted signal is random binary number $\mathrm{I}(\mathrm{t})$ Figure 5 . The transmitted signal is added to the generated chaotic y signal shown in Figure.6, and the $S(t)=y+I(t)$ is feed into the receiver Figure.7. The chaotic Yr signal is regenerated allowing a single subtraction to retrieve the transmitted signal, [Y+I (t)]-Yr $=\mathrm{I}(\mathrm{t})]$, If $\mathrm{Y}$ $=$ Yr. This is a result of synchronization as in Figure. 8 which shows the information binary signal retrieved at receiver output of scope. Figure. 9 shows the error between the information signal transmitted and recovered.

\section{CONCULSION}

This paper focuses on the Lorentz Attractor's chaotic oscillator circuits [10] and their applications in signal masking communications. Lorentz Attractor's chaotic oscillator circuits were designed and simulated. Chaotic signal masking circuits were realized using Matlab-Simulink and related figures $(3,9)$ point out that Matlab-Simulink output proves the same conclusions. We have demonstrated in simulations that chaos can be 
synchronized and applied to secure communications. We suggest that this phenomenon of chaos synchronism may serve as the basis for little known Lorentz Attractor to achieve secure communication. Simulation results are used to visualize and illustrate the effectiveness of Lorentz chaotic system in signal masking [9]. Figure.5 modulated Signal also shows that is becomes difficult for eye dropper or hacker to distinguish between the information containing signal and noise, which makes this system more secure .All simulations results performed on Lorentz chaotic system are verified the applicable of secure communication [11].

\section{ACKNOWLEDGEMENT}

I would like to thank the respected Dr. Udhav Bhosle and Prof. Sanjay D. Deshmukh for supporting and giving immense guidance.

\section{REFERENCES}

[1] Oppenheim, A.V. \& Cuomo, K.M. "Chaotic Signals and Signal Processing” Digital Signal Processing Handbook Ed. Vijay K. Madisetti and Douglas B. Williams Boca Raton: CRC Press LLC, 1999

[2] L. M. Pecora and T. L. Carroll, "Synchronization in chaotic systems", Phys. Rev. Lett, 64(8):821-5, 1990.

[3] Ihsan PEHLIVAN,, Yılmaz UYAROGLU, M. Ali YALÇIN ve Selçuk COSKUN "Design and Simulations of the Arneodo Attractor's Chaotic Oscillator and Signal Masking Circuits", 5th International Advanced Technologies Symposium (IATS'09), May 13-15, 2009, Karabuk, Turkey

[4] Sneha Venkateswar, Gargi Rajadhyaksha, Jinal Shah "Analysis Of Chaotic Signals As An Alternative To Pseudo-Random Sequences In Ds-Cdma"Canadian Journal on Computing in Mathematics, Natural Sciences, Engineering and Medicine Vol. 4 No. 2, April 2013

[5] Taejoo Chang, Iickho Song, Jinso Bae, Hong-Gil Kim "Chaotic Signal masking based on Lorentz System"

[6] Jing Pan, Qun Ding, And Bao Xiang Du "A New Improved Scheme of Chaotic Making Scheme Secure Communication Based on Lorentz System”, Int. J. Bifurcation Chaos 22, 1250125 (2012)]

[7] http://en.wikipedia.org/wiki/Lorenz_attractor, "Lorenz Attractor," Wikipedia, the free encyclopedia, 2008.

[8] L. M. Pecora and T. L. Carroll, "Synchronization in Chaotic Systems" Phy. Rev. Lett., vol. Vol 64, pp. pp. 821-824, 1990.

[9] Nguyen Xuan Quyen, Vu Van Yem, and Thang Manh Hoang, "A Chaos-Based Secure Direct Sequence/Spread-Spectrum Communication System", Hindawi Publishing Corporation Abstract and Applied Analysis Volume 2013, Article ID 764341, 11 pages http://dx.doi.org/10.1155/2013/764341

[10] E. N. Lorenz, Deterministic non-periodic flow, J. Atmos. Sci., 20, 130-141, 1963

[11] I. Pehlivan and Y.Uyaroğlu, "Simplified chaotic diffusionless Lorentz attractor and its application to secure communication systems", IET Commun., 2007, 1(5):1015-1022, 2007.

[12] Robert L. V. Taylor, Advised by Dr. John David, “Attractors: Nonstrange to Chaotic”. 